\title{
Optimized CMOS photodetector structures for the detection of green luminescent probes in biological applications
}

\author{
R.A. Yotter*, M.R. Warren, D.M. Wilson \\ Department of Electrical Engineering, University of Washington, P.O. Box 352500, Seattle, WA 98195-2500, USA \\ Received 3 October 2003; received in revised form 7 April 2004; accepted 13 April 2004 \\ Available online 28 May 2004
}

\begin{abstract}
Recent developments in lab-on-a-chip applications have increased the need for low-power, small-footprint photodetectors to detect luminescent reporters used to monitor biological events. Presented is a CMOS photodiode structure that is optimized to detect green light, the peak emission wavelength of commonly used fluorophores and other luminescent probes. The photodiode geometry uses a lightly doped striped cathode geometry to maximize the depletion region in areas of highest light absorbance, increasing the sensitivity to green light. The devices, realized in $1.5 \mu \mathrm{m}$ CMOS technology, have up to a $63 \%$ increased signal-to-noise response for green light compared to blue-enhanced CMOS photodiode structures. These devices also generate an output 4.5 times larger than conventional CMOS detectors for detecting fluorescent emissions from a $1 \mu \mathrm{M}$ fluorescein solution. The devices are designed to be integrated in total analysis systems which rely on the detection of fluorecent or phosphorescent reporters, and which are compact, low-power, and low-cost.
\end{abstract}

(C) 2004 Elsevier B.V. All rights reserved.

Keywords: Photodetector; Photodiode; Stripe-shaped photodiode; CMOS; Fluorescence detector

\section{Introduction}

Fluorescent and phosphorescent probes are widely used in applications for detecting biological events. Recent developments in microfabrication and MEMS have generated a need for small, low-cost, and low-power photodetectors that are capable of detecting emissions from luminescent reporters in automated processes. Applications include laboratory equipment which automates routine biological experiments, such as DNA sequencing [1-5] and capillary electrophoresis [6,7]; portable hand-held instrumentation [8]; and new detectors to sense single cells, such as life-on-a-chip devices [9]. For all of these applications, the characteristics of the luminescent probe must match the detection capabilities of the photodetector. A review of photodetector designs, performance metrics, and matching of metrics to the detection of biological reporters can be found in [10]. Photodevices with enhanced response to blue light have been developed [11,12], and integrating circuit schemes have successfully been applied to the detection of bioluminescence [13-15]. Since most fluorescent probes

\footnotetext{
* Corresponding author. Tel.: +1-2065432505; fax: +1-2065433842. E-mail address: raytrace@ee.washington.edu (R.A. Yotter).
}

emit green light, whether the probe detects DNA [16], $\mathrm{pH}$ [17], or cell viability [18], a small, low-cost, low-power photodetector optimized for green light is needed for these applications.

In this paper, a CMOS photodetector optimized for detecting green light is presented. The photodiode structure uses interdigitated n-well fingers to increase the depletion region in areas of maximal absorption of green light, thereby increasing the detector's overall sensitivity and response to green light. For a silicon device, photons at shorter wavelengths are absorbed near the surface (corresponding to blue or ultraviolet light), and photons at longer wavelengths are absorbed deeper within the substrate (corresponding to red light). Green light is absorbed both near the surface and deeper within the substrate; the new interdigitated n-well structure places the depletion region within the maximum absorption area for green light. An n-well structure used in this photodetector design offers the additional benefit of an increased depletion region width, because n-well doping is relatively light. The sensitivity is further enhanced from the Gaussian doping profile of the n-well, which creates a near-intrinsic region within the junction (analogous to a pin junction). Experimental results clearly show that the new CMOS photodetector structure has an increased sensitivity and detection limit for green light. 
The CMOS photodetector can be integrated in a total analysis system that is compact, low-power, and low-cost, while the superior reporter detection capabilities of this photodiode will enable higher sensitivity for the system. This detector is ideal for use in biological life-on-a-chip applications that rely on detection of green fluorescent or phosphorescent reporters.

\section{Theory of operation}

Four photodiode structures were fabricated using the AMI $1.5 \mu \mathrm{m}$ CMOS process. To determine the best geometry for the detector, the interdigitated photodiode structures are designed with different finger width $W$ and finger spacing $D$ (Fig. 1). Two of the photodetector structures are designed for enhanced sensitivity to green light by using interdigitated n-well fingers to create the photosensitive depletion region. The geometries for these two structures are $(W=$ $6.4 \mu \mathrm{m}, D=4.8 \mu \mathrm{m})$ and $(W=8 \mu \mathrm{m}, D=6.4 \mu \mathrm{m})$. The other two structures are blue-enhanced photodiodes fabricated as described in [11], with a geometry designed for operation at $5 \mathrm{~V}$ reverse bias and minimization of dead area for the AMI $1.5 \mu \mathrm{m}$ process. Dead area includes all regions that are not depleted and thus do not generate a significant photo-induced current in response to light. To minimize dead area for the blue-enhanced photodiode structures, the fingers should be as narrow as possible within the constraints of the design rules for the fabrication process. The geometries for the blue-enhanced photodiodes were $(W=3.2 \mu \mathrm{m}$, $D=3.2 \mu \mathrm{m})$ and $(W=2.4 \mu \mathrm{m}, D=3.2 \mu \mathrm{m})$. Both blue-enhanced structures include a planar n-well/p-substrate photodiode deep within the substrate, which increased their sensitivity to light at longer wavelengths. This geometry was the best possible blue-enhanced structure for maximizing sensitivity. All photodiode structures occupied approximately the same lateral area, and three structures had approximately the same active area. A planar photodiode was also fabricated to ensure performance enhancement over the most basic CMOS photodetector structure.

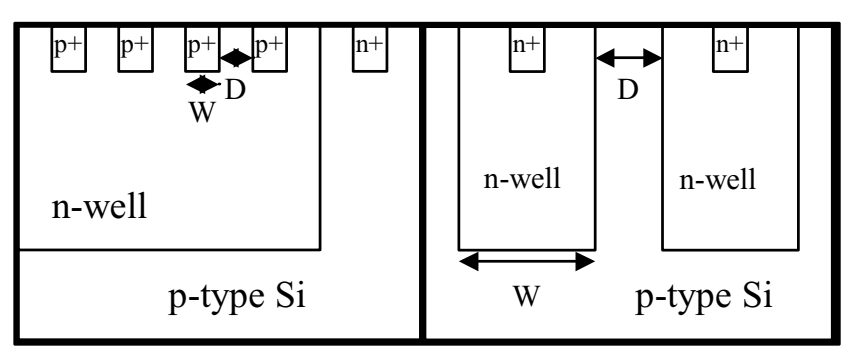

Fig. 1. The cross-sectional geometry of the blue-enhanced structure (left) and the geometry of the green-enhanced structure (right) highlights the differences between the two structures. The blue-enhanced structure uses interdigitated $\mathrm{p}+$-diffusion fingers to increase the depletion region area near the surface of the detector, while the green-enhanced structure uses n-well fingers to increase the depletion region slightly deeper within the substrate.
Photodetectors fabricated with CMOS technology usually have non-uniform doping profiles, which creates potential barriers within the structure. Pauchard, et al. reported two potential barriers, one arising from a depletion of donor atoms from the surface of the device, another arising from non-uniform n-well doping [11]. Thus, $\mathrm{p}+-\mathrm{n}$-well photodiodes have a dead layer at the surface of the device due to the $p+$ doping. By eliminating $p+$ regions from the device, there is no potential barrier introduced at the surface of the fingers, which sin turn increases the potential sensitivity of the device to blue and green light.

A small potential barrier may remain due to the non-uniform doping profile of the n-well region, but this region only exists at the fingers for the green-enhanced structures. Regions between the fingers do not have any potential barrier at all, since the regions consist of p-substrate, which has very uniform doping. Also, the AMI $1.5 \mu \mathrm{m}$ process features a $4 \mu \mathrm{m}$ n-well junction depth (compared to a $1.5 \mu \mathrm{m}$ junction depth reported by Pauchard et al.), which extends the sensitive region deeper into the substrate, thereby enhancing sensitivity to blue and green light even further.

The equations for the responsivity of the blue-enhanced structures have been derived previously [11], and the equation is reproduced here for convenience. For a striped photodiode structure, the responsivity is

$$
\begin{aligned}
R(\lambda)= & \frac{q \eta(\lambda)}{h v} T(\lambda)\left\{\frac{W}{W+D}\left[s \int_{0}^{X_{\mathrm{D}}} \mathrm{e}^{-\alpha(\lambda) x} \mathrm{~d} x+\int_{X_{\mathrm{D}}}^{X_{\mathrm{B}}} \mathrm{e}^{-\alpha(\lambda) x} \mathrm{~d} x\right]\right. \\
& \left.+\frac{D}{W+D}\left[(1-c) \int_{0}^{X_{\mathrm{B}}} \mathrm{e}^{-\alpha(\lambda) x} \mathrm{~d} x\right]\right\}
\end{aligned}
$$

Here, $q$ is the charge of an electron, $\eta$ the internal quantum efficiency, $h$ Planck's constant, $v$ the frequency of the photon, $T$ the surface transmission coefficient, $W$ the width of the fingers, $D$ the distance between fingers, $s$ the percentage of minority carriers absorbed in the dead layer, $\alpha$ the absorption coefficient, $X_{\mathrm{B}}$ the potential barrier depth of the n-well, $X_{\mathrm{D}}$ the dead layer width, and $c$ the percentage of generated carriers between stripes that do not reach the anode.

The responsivity increase for the green-enhanced photodiodes arises from the value of $s$ for the two types of structures. Since there is no $\mathrm{p}+$ diffusion layer in the green-enhanced structure, no dead layer exists and $s=1$. For the blue-enhanced structures, $s<1$ because of the presence of the $\mathrm{p}+$ diffusion layer. Another difference between the blue- and green-enhanced photodiode structures is the penetration depth of the photons. While blue-enhanced photodiodes must maximize the active area near the surface of the structure, green-enhanced photodiodes need to maximize the active area within a region that cannot be depleted from either a planar $\mathrm{p}+$-n-well diode or a n-well-psubstrate diode.

One calculation of interest is the ratio of responsivities for the blue- and green-enhanced photodiode structures. Assuming that the wavelength is monochromatic (so $\alpha$ is constant), and the depth of the potential barrier $X_{\mathrm{B}}$ is the same 
for both structures, the responsivity ratio at one wavelength can be reduced to

$$
\frac{R_{\mathrm{G}}}{R_{\mathrm{B}}}=\frac{1}{\left(s^{\prime} W_{\mathrm{B}}+D_{\mathrm{B}} / W_{\mathrm{B}}+D_{\mathrm{B}}\right)}>1
$$

where $s^{\prime}(<1)$ is a correction factor that arises from a non-unity $s$ value for the blue-enhanced structures due to the $\mathrm{p}+$ potential barrier. The responsivity increase using this analysis is always greater than unity. This calculation assumes that active area is maximized throughout the full depth between the fingers $(c=0)$. This assumption may not be valid near the surface and deep within the photodetector structure where the doping levels may be lower than predicted, which could decrease the responsivity of the structure to blue and red light.

\section{Simulation}

The photodiode structures have been simulated using the LUMINOUS package in ATLAS (Fig. 2). This program estimated the responsivity and dark current of a photodiode structures, and these values were used to calculate the SNR.

In the LUMINOUS package, the generation of electronhole pairs, designated by $G$, is modeled using the following formula:

$G=\eta \frac{P \lambda}{h c} \alpha \mathrm{e}^{-\alpha z}$ where $\eta$ is the internal quantum efficiency, $h$ Planck's constant, $P$ the cumulative effects of reflections, transmissions, and loss due to absorption, $\lambda$ the wavelength of the photons, $\alpha$ the absorption coefficient, $c$ the speed of light, and $z$ the distance into the substrate. The responsivity is then calculated by measuring the subsequent photogenerated current and dividing by the power emitted by the simulated light source (in Watts).

The photodiode structures were equivalent in structure to those outlined above. The model assumed an n-well junction depth of $4.0 \mu \mathrm{m}$, a $\mathrm{p}+$ diffusion junction depth of $0.2 \mu \mathrm{m}$, an n-well doping of $10^{17} \mathrm{~cm}^{-3}$, a $\mathrm{p}+$ diffusion doping concentration of $10^{20} \mathrm{~cm}^{-3}$, and a Gaussian doping profile at junction interfaces. The absorption coefficient of silicon was the default value for the software package.

The simulated light source scanned through the wavelengths of interest at a known intensity, and the absorption coefficient of silicon is used to calculate the generated photocurrent. The dark current, or noise, was calculated by simulating the non-illuminated structure to obtain an estimate of the dark current. The signal-to-noise ratio was then calculated by first calculating the total current in response to the impinging light, then dividing by the dark current.

The simulation results indicate that the SNR for the green-enhanced ( $W=8 \mu \mathrm{m}, D=6.4 \mu \mathrm{m})$ structure in-crease by up to $20 \%$ (at wavelength of $500 \mathrm{~nm}$ ) compared to the blue-enhanced photodetector structures. The simulated and experimental results differed due to three

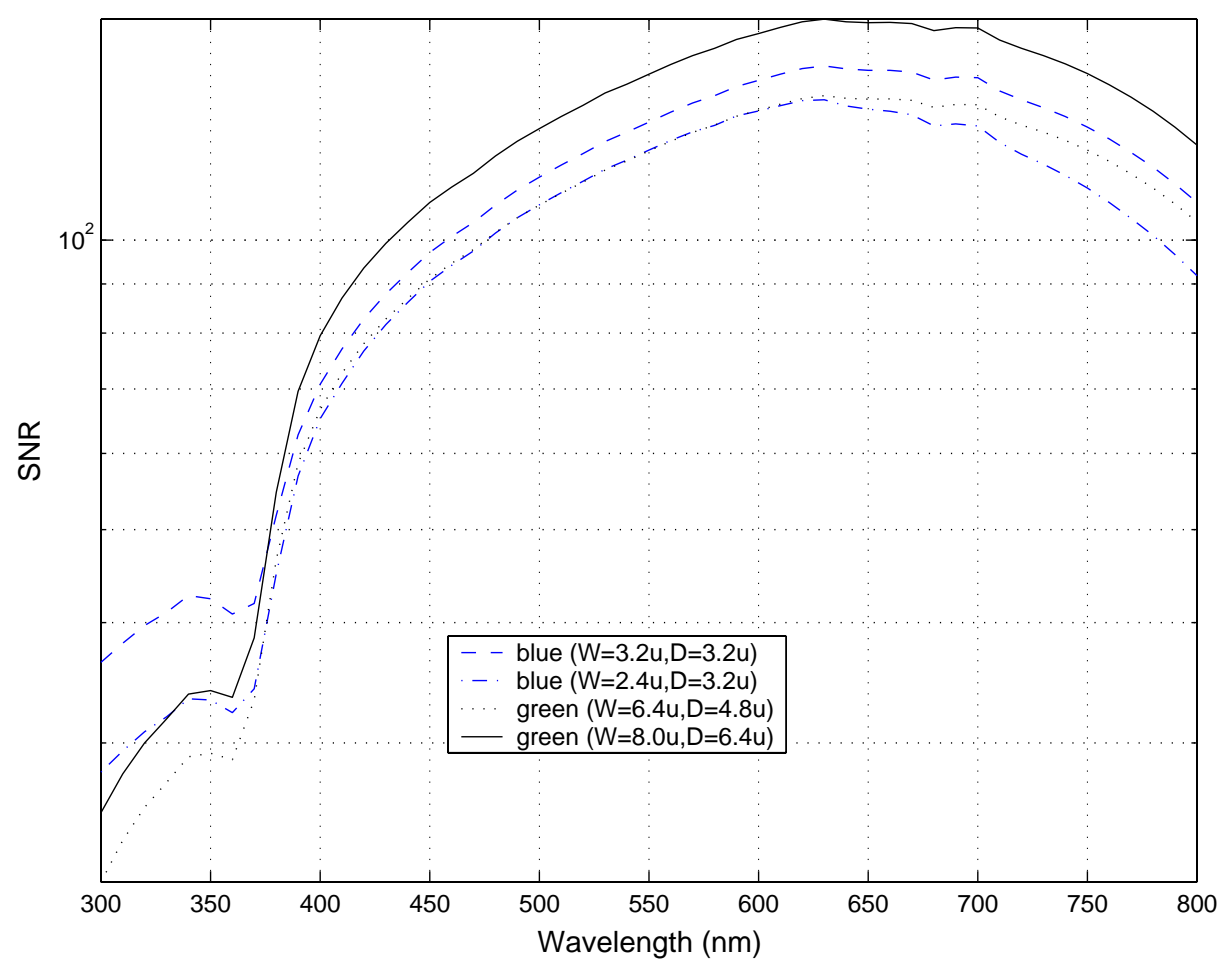

Fig. 2. The performance of the photodiodes was simulated using the LUMINOUS package in ATLAS. The simulated optical intensity was $1 \mathrm{~mW}^{-2}$, and the noise current was extracted from the $I-V$ curves under no-light conditions. Although simulation of the blue-enhanced structure indicated that it would be more sensitive to blue light, the green-enhanced structure performs better for green, yellow, and red wavelengths. 
effects: (1) the nonlinear absorption spectrum of the silicon nitride passivation layer are not simulated; and (2) the metal connections to fingers did not reduce the active area in the simulations.

\section{Experimental}

Four photodetector structures have been fabricated: two green-enhanced structures with ( $W=6.4 \mu \mathrm{m}, D=4.8 \mu \mathrm{m}$ ) and $(W=8 \mu \mathrm{m}, D=6.4 \mu \mathrm{m})$, and two blue-enhanced structures with $(W=3.2 \mu \mathrm{m}, D=3.2 \mu \mathrm{m})$ and $(W=$ $2.4 \mu \mathrm{m}, D=3.2 \mu \mathrm{m})$. Two CMOS chips were fabricated using the MOSIS foundry service. One chip has an intact silicon nitride passivation layer on top of the photodetector structures, reducing the efficiency of the photodetector structures to detect blue light. The silicon nitride passivation layer effectively attenuates the intensity of the incident light. The second chip uses the GLASS design layer to remove silicon nitride from the active area of the photodetectors. When regions are highlighted with the GLASS layer in a CMOS layout program, the MOSIS foundry service translates this information into a physical etch of the passivation nitride layer. This layer is most commonly used to expose the metal bond pads at the periphery of the chip. The signal-to-noise ratio is measured from 300 to $800 \mathrm{~nm}$ using a Perkin-Elmer LS50B Luminescence Spectrometer as the light source, with excitation slits of either 5 or $10 \mathrm{~nm}$ depending on the test. The chip is mounted so that the light from the Spectrometer fully illuminates the chip surface, and the electrical response

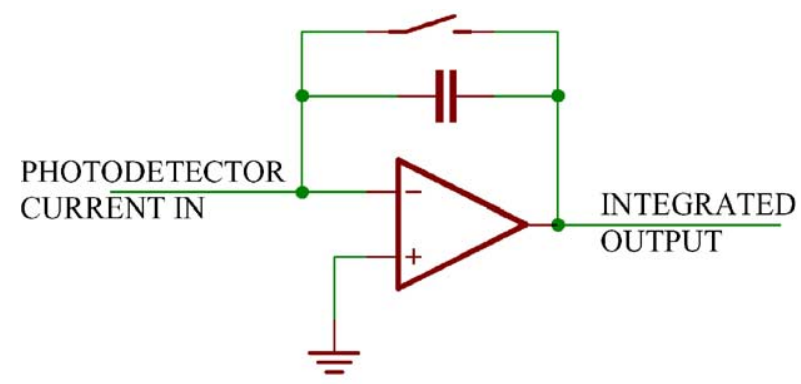

Fig. 3. An integrating luminescent circuit was used to amplify the photodetector signal. Current from photodetector is collected by the capacitor. The circuit is reset using a switch placed in parallel to the capacitor, which discharges the stored charge on the capacitor.

is measured using a Keithley 236 Source Measurement Unit.

The $1 \mu \mathrm{M}$ fluorescein solution used for testing all four structures is prepared using fluorescein-5-isothiocyanate (Molecular Probes, Eugene, OR) dissolved in a $0.1 \mathrm{M}$ bicarbonate solution. Fluorescein is placed in a fiber optic fluorescence spectroscopy system, which includes a $1 \mathrm{~cm}$ CVD-UV cuvette held in a CUV-ALL-UV four-way cuvette holder, light is generated from the LS-1 Tungsten Halogen Light Source, and components are interconnected with P-1000-2-UV-Vis fiber optic cables (Ocean Optics, Dunedin, FL). The excitation and emission filters are obtained from Omega Optical (XF115-2, Omega Optical Inc., Brattleboro, VT). One end of a fiber optic cable is placed within $1 \mathrm{~cm}$ of the surface of the CMOS chip, and the

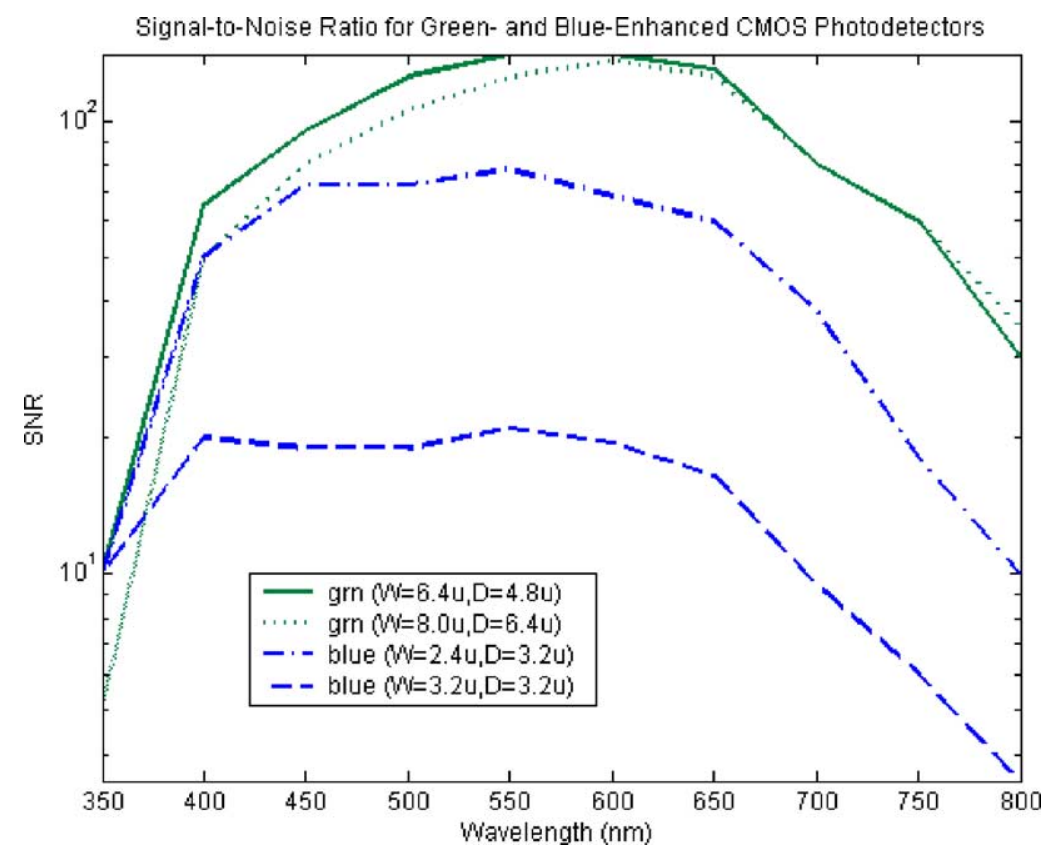

Fig. 4. The experimental results of photodiode signal-to-noise ratio (SNR) demonstrate the improved SNR of the green-enhanced structures. For these results, the silicon nitride layer is still intact, which lowers the possible SNR at shorter wavelengths for all photodiodes. The response is measured every $50 \mathrm{~nm}$ with a $10 \mathrm{~nm}$ optical excitation slit (optical bandwidth). The active area of the blue-enhanced ( $W=2.4 \mu \mathrm{m}, D=3.2 \mu \mathrm{m})$ structure is slightly larger than other photodetectors, which increases its apparent SNR. 
response of all photodiode structures is measured without changing the alignment between tests. A bioluminescent sensing circuit is used to integrate the signal, shown in Fig. 3 [13].

\section{Results and discussion}

The signal-to-noise ratio (SNR) is measured to determine the efficiency of the photodetector for detecting green light.

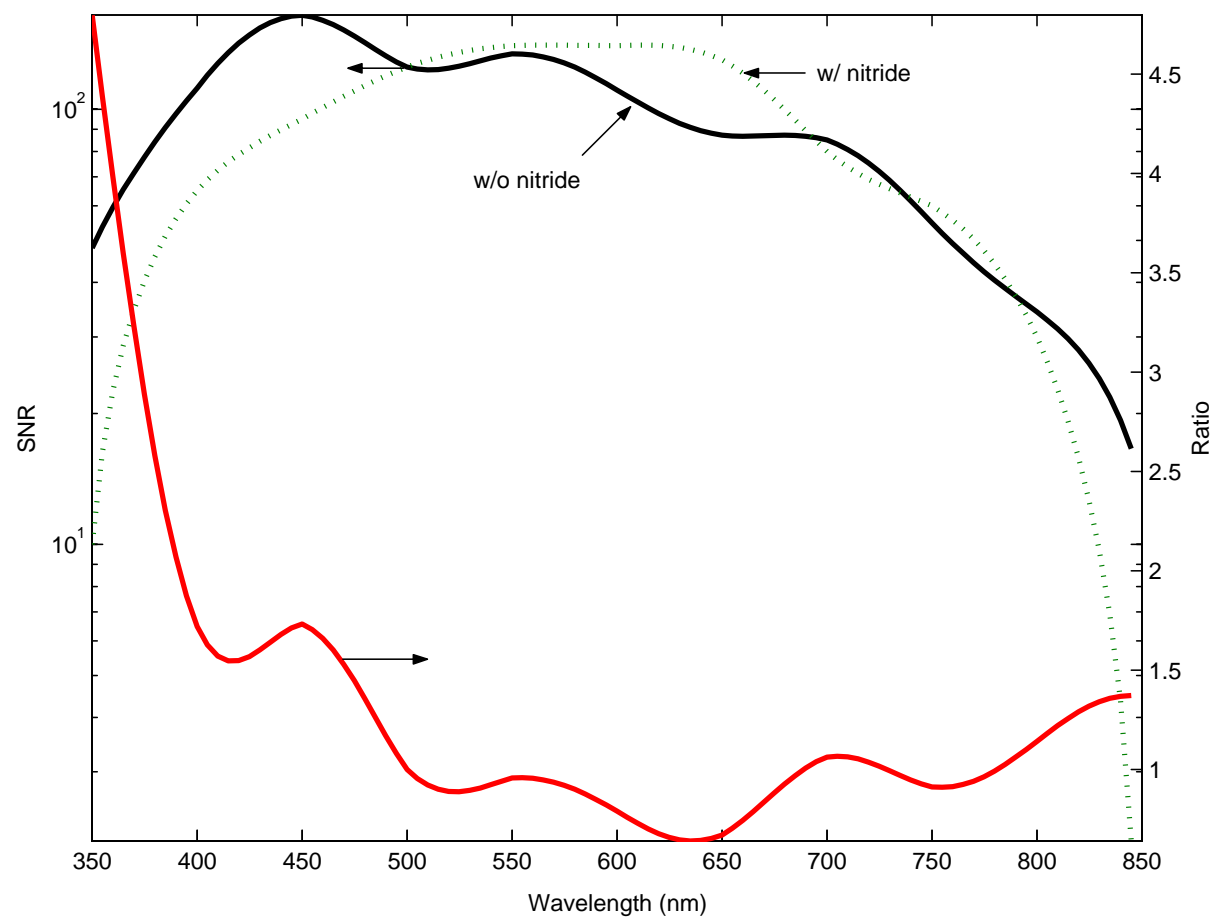

Fig. 5. The removal of silicon nitride improved the sensitivity of the photodiode structure at short wavelengths (which corresponds to blue light). The data shown are for the green-enhanced photodiode structure $(W=6.4 \mu \mathrm{m}, D=4.8 \mu \mathrm{m})$.

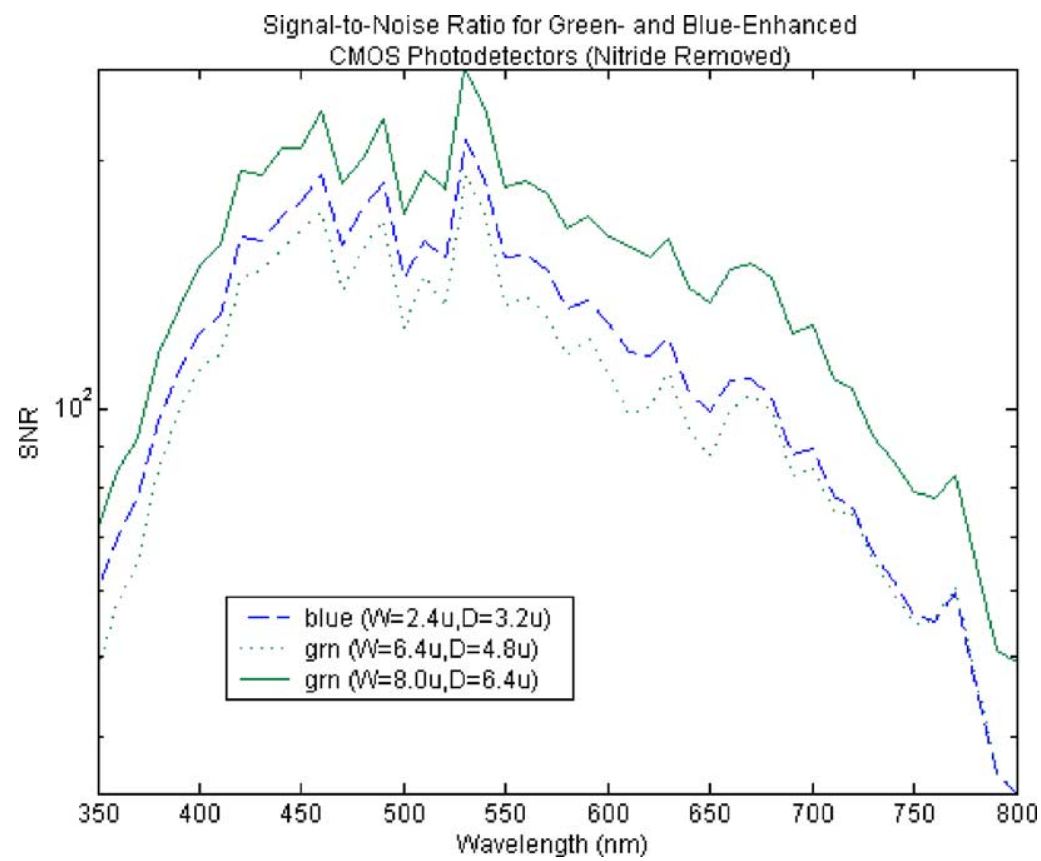

Fig. 6. Removal of the silicon nitride layer increased the SNR at shorter wavelengths (blue light). The SNR for the green-enhanced structures was higher over the entire wavelength range compared to the blue-enhanced structure. The performance difference at short wavelengths was smaller, which was expected based on simulation results. The excitation slit was $5 \mathrm{~nm}$ with a $10 \mathrm{~nm}$ optical excitation slit (optical bandwidth). 
To calculate the signal-to-noise ratio, the dark current of the non-illuminated photodiode structure was first measured. Then the structure was illuminated with light at a specific wavelength, and the resulting current was measured. The SNR was calculated by dividing the current response of the illuminated structure with the dark current. The SNR is the more appropriate metric for photodiode structures targeted for applications in which the light level is very low, since the SNR indicates the lowest possible detection limit of the photodiode structure.

Experimental results for photodetectors with an intact silicon nitride layer demonstrate that the new photodiode structure increases the SNR by $40 \%$ for green light compared to other CMOS photodiode structures (Fig. 4). The green-enhanced structures ware more responsive to green light than the blue-enhanced photodetector structures. Because the silicon nitride passivation layer is not removed in this intial design, however, the SNR for blue light was limited for all structures. Removal of the silicon nitride increased the SNR for blue light (Fig. 5). It also increased the SNR of the structure to green light and red light.

A summary of photodetector performance with the silicon nitride removed is shown in Fig. 6. Only the best response from a blue-enhanced structure is shown. For green light $(500 \mathrm{~nm})$, the green-enhanced structure $(W=$ $8.0 \mu \mathrm{m}, D=6.4 \mu \mathrm{m})$ had a $22 \%$ increase compared to the best-performing blue-enhanced structure ( $W=2.4 \mu \mathrm{m}$, $D=3.2 \mu \mathrm{m}$ ), and a $63 \%$ increase compared to the other blue-enhanced structure ( $W=3.2 \mu \mathrm{m}, D=3.2 \mu \mathrm{m})$. The ratio of the SNR for the green-enhanced structure
Table 1

Photodiode response to $1 \mu \mathrm{M}$ fluorescein was measured using an integrating circuit as described in [2]

\begin{tabular}{lll}
\hline CMOS photodiode & $I_{\mathrm{F}}(\mathrm{pA})$ & SNR \\
\hline Green $(W=6.4 \mu \mathrm{m}, D=4.8 \mu \mathrm{m})$ & 0.225 & 1.10 \\
Green $(W=8 \mu \mathrm{m}, D=64 \mu \mathrm{m})$ & 0.175 & 1.07 \\
Blue $(W=3.2 \mu \mathrm{m}, D=32 \mu \mathrm{m})$ & 0.075 & 1.05 \\
Blue $(W=2.4 \mu \mathrm{m}, D=3.2 \mu \mathrm{m})$ & 0.05 & 1.02 \\
\hline
\end{tabular}

The signal $I_{\mathrm{F}}$ was determined by measuring the difference between the response in the presence and absence of the fluorescent signal. The optical train included excitation and emission filters for the biological reporter. Because the optical signal intensity is low, the SNR is near threshold for the detectors. Low optical signal intensity was the result of inefficient coupling between optical elements.

compared to the best blue-enhanced structure is shown in Fig. 7 on a logarithmic scale. This clearly illustrates that although green-enhanced structure is only slightly more sensitive to blue light, it outperforms the blue-enhanced structures for green, yellow, and orange light. This structure is more sensitive to light emitted from luminescent probes.

The detection of fluorescence from a biological reporter has also been measured using those photodetector structures whose silicon nitride is still intact. The photodetectors are used to measure the fluorescence from $1 \mu \mathrm{M}$ fluorescein, and the signal is integrated using an integrating circuit similar to the gated integrator used by Simpson et al. to measure bioluminescence [13]. The results (shown in Table 1) demonstrate that the green-enhanced structures could generate a signal that is 4.5 times larger than the blue-enhanced photodetector

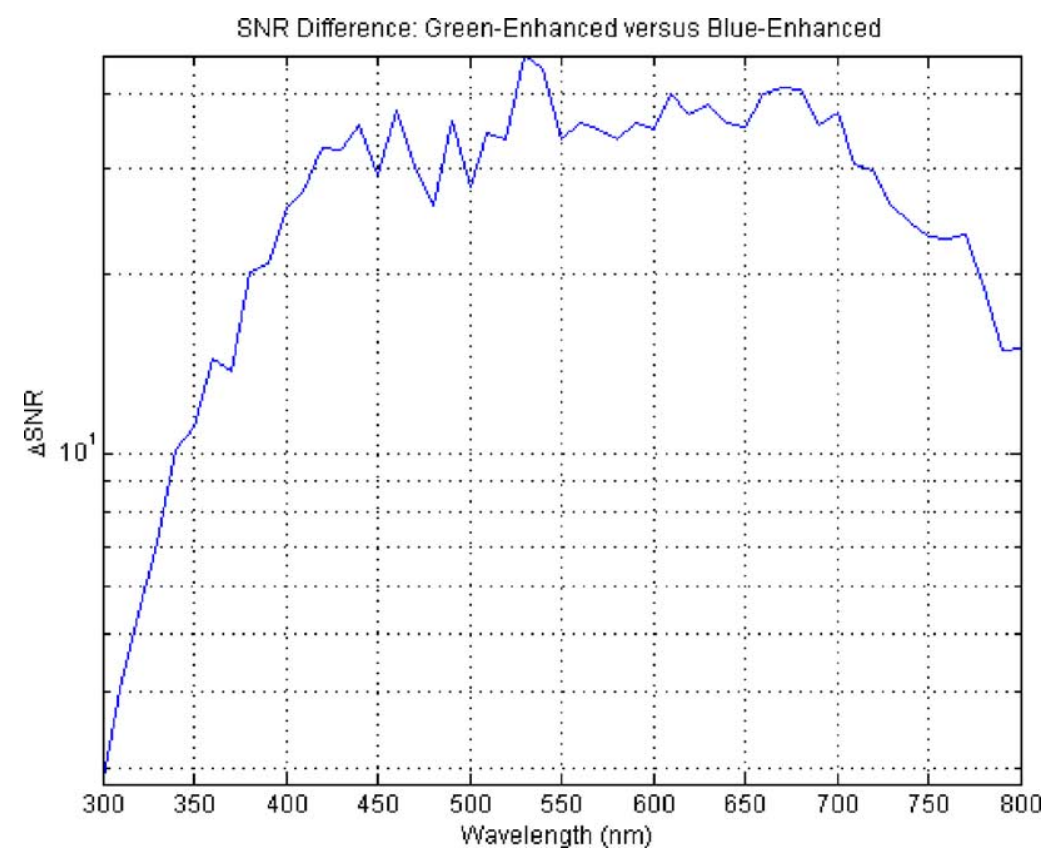

Fig. 7. The SNR difference between top-performing blue- and green-enhanced structures is largest for longer wavelengths, especially wavelengths at which luminescent probes emit. There is an overall improvement in SNR over the entire visible light range, and the performance difference was smallest for short-wavelength light. 
structures. Several stages along the optical train were poorly coupled, which resulted in large losses of the light signal. The detection limit could be dramatically improved by designing an optical train with better optical coupling. With the optical setup used in this system, the lowest detectable fluorescein concentration is approximately $36 \mathrm{nM}$. To detect the lower-intensity signal, a much longer integration time would be needed.

\section{Conclusions}

A new photodetector structure optimized for the detection of green light has been realized using a standard CMOS AMI $1.5 \mu \mathrm{m}$ process. Optimized detection of green light is achieved by using an interdigitated n-well finger structure, which increased the depletion region in regions of maximal absorption of green light. Other advantages of the structure include light doping to increase the depletion region width, and a graded or Gaussian doping profile at the n-well-p-substrate junction, which creates a nearly intrinsic region within the junction. This intrinsic region increases the photo-sensitive region of the detector. The new photodetector structures have an SNR increase up to $63 \%$ for detecting green light at $500 \mathrm{~nm}$. In combination with an integrating bioluminescent circuit, the photodetector has been demonstrated to detect fluorescein, a common biological reporter, with higher efficiency than other CMOS photodetector structures. The output signal from the green-enhanced structures was 4.5 times larger than for other structures, and this could be improved on further through the development of small-footprint optical coupling components and the use of an anti-reflective coating.

Since most biological luminescent reporters emit green light, the new photodetector structure is ideal for applications that require an inexpensive, low-power, and small-footprint photodetection solution. Since the photodetector was built using a standard CMOS process, the detector is also capable of being easily integrated with signal processing circuitry to perform a dedicated measurement task. Potential applications include integration with laboratory equipment that automates routine biological experiments, portable hand-held instrumentation, and life-on-a-chip devices for detecting single cells.

\section{Acknowledgements}

Funding for this research was provided by the NIH National Human Genome Research Institute Centers of Excellence in Genomic Science, the Microscale Life Sciences Center at the University of Washington, grant number 1 P50 HG002360, "CEGSTech: Integrated Biologically Active Microsystems".

\section{References}

[1] D.R. Meldrum, S.E. Moody, Automating high-throughput fluid sample handling for biotechnology and chemistry, in: Proceedings of the IEEE International Conference on Robotics \& Automation, San Francisco, CA, 2000, 198-205.

[2] D.R. Meldrum, W.H. Pence, S.E. Moody, D.L. Cunningham, M. Holl, P.J. Wiktor, Automated Microfluidics for Genomics, in: Proceedings of the 23rd Annual EMBS International Conference, Istanbul, Turkey, 25-28 October 2001, pp. 2923-2926.

[3] M.A. Burns, B.N. Johnson, S.N. Brahmasandra, K. Handique, J.R. Webster, M. Krishnan, T.S. Sammarco, P.F. Man, D.K. Jones, D. Heldsinger, C.H. Mastrangelo, D.T. Burke, An integrated nanoliter DNA analysis device, Science 282 (1998) 484-487.

[4] P.R. Selvaganapathy, E.T. Carlen, C.H. Mastrangelo, Recent progress in microfluidic devices for nucleic acid and antibody assays, Proc. IEEE 91 (2003) 954-975.

[5] C.H. Mastrangelo, M.A. Burns, D.T. Burke, Microfabricated devices for genetic diagnostics, Proc. IEEE 86 (1998) 1769-1787.

[6] J.R. Webster, C.H. Mastrangelo, Large-volume integrated capillary electrophoresis stage fabricated using micromachining of plastics on silicon substrates, in: TRANSDUCERS '97, Chicago, IL, 1997, pp. 503-506.

[7] K.D. Kramer, K.W. Oh, C.H. Ahn, J.J. Bao, K.R. Wehmeyer, Optical MEMS-based fluorescence detection scheme with applications to capillary electrophoresis, in: Proceedings of the SPIE-Conference on Microfluidic Development Systems, Santa Clara, CA, 1998, pp. $76-85$.

[8] D.M. Wilson, S. Hoyt, J. Janata, K. Booksh, L. Obando, Chemical sensors for portable, handheld field instruments, IEEE Sens. J. 1 (2001) 256-274.

[9] D. Meldrum, M. Holl, P. Seriburi, S. Phillips, S.H. Chao, L. Lang, F. Kosar, MEMS modules for life-on-a-chip, in: Proceedings of the 2003 International Symposium Circuits Systems, ISCAS, Bangkok, Thailand, 2003, pp. 638-641.

[10] R.A. Yotter, D.M. Wilson, A review of photodetectors for sensing light-emitting reporters in biological systems, IEEE Sens. J. 3 (2003) 288-303.

[11] A. Pauchard, P.-A. Besse, R.S. Popovic, A silicon blue/UV selective stripe-shaped photodiode, Sens. Actuators A 76 (1999) 172-177.

[12] C.S. Yin, The p-i-n junction-surface depletion-layer photodiode, IEEE Elect. Dev. Lett. 12 (1991) 442-443.

[13] M.L. Simpson, G.S. Sayler, B.M. Applegate, S. Ripp, D.E. Nivens, M.J. Paulus, G.E. Jellison Jr., Bioluminescent-bioreporter integrated circuits form novel whole-cell biosensors, Trends Biotechnol. 16 (1998) 332-338.

[14] M.L. Simpson, G.S. Sayler, G. Patterson, D.E. Nivens, E.K. Bolton, J.M. Rochelle, J.C. Arnott, B.M. Applegate, S. Ripp, M.A. Guillorn, An integrated CMOS microluminometer for low-level luminescence sensing in the bioluminescence bioreporter integrated circuit, Sens. Actuators B 72 (2001) 134-140.

[15] E.K. Bolton, G.S. Sayler, D.E. Nivens, J.M. Rochelle, S. Ripp, M.L. Simpson, Integrated CMOS photodetectors and signal processing for very low-level chemical sensing with the bioluminescent bioreporter integrated circuit, Sens. Actuators 85 (2002) 179-185.

[16] Molecular Probes Inc. (Online). Data Table-8.1 Nucleic Acid Stains. Available: http://www.probes.com/servlets/datatable?id=32237. 10 September 2003 (date accessed).

[17] Molecular Probes Inc. (Online). Data Table-21.2 Probes Useful at Near-Neutral pH. Available: http://www.probes.com/servlets/ datatable?id=28859. 10 September 2003 (date accessed).

[18] Molecular Probes Inc. (Online). Data Table-15.2 Viability and Cytotoxicity Assay Reagents. Available: http://www.probes.com/ servlets/datatable?id=29279. 10 September 2003 (date accessed). 